Title:

\section{APT LLRF CONTROL SYSTEM MODEL RESULTS}

Submitted to:

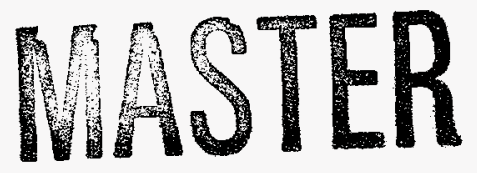

Author(s):

\section{Amy H. Regan, LANSCE-5 \\ C. Ziomek, B\&B Technologies,} Albuquerque, NM

\section{RECEIVED}

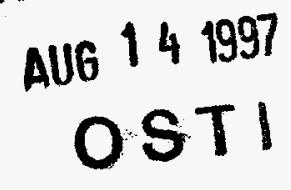

\section{Particle Accelerator Conference Vancouver, CANADA May 12-15, 1997}

\title{
DISCLAIMER
}

This report was prepared as an account of work sponsored by an agency of the United States Government. Neither the United States Government nor any agency thereof, nor any of their employees, makes any warranty, express or implied, or assumes any legal liability or responsibility for the accuracy, completeness, or usefulness of any information, apparatus, product, or process disclosed, or represents that its use would not infringe privately owned rights. Reference herein to any specific commercial product, process, or service by trade name, trademark, manufacturer, or otherwise does not necessarily constitute or imply its endorsement, recommendation, or favoring by the United States Government or any agency thereof. The views and opinions of authors expressed herein do not necessarily state or reflect those of the United States Government or any agency thereof.

\section{Los Alamos}

NATIONAL LABORATORY

Los Alamos National Laboratory, an effirmative action/equal opportunity employer, is operated by the University of California for the U.S. Department of Energy under contract W-7405-ENG-36. By acceptance of this articte, the publisher recognizes that the U.S. Government retains a nonexclusive, royalty-free license to publish or reproduce the published form of this contribution, or to allow others to do so, for U.S. Government purposes. Los Alamos National Laboratory requests that the publisher identify this article as work performed under the auspices of the U.S. Department of Energy. The Los Alamos National Laboratory strongly supports academic freedom and a researcher's right to publish; as an institution, however, the Laboratory does not endorse the viewpoint of a publication or guarantee its technical correctness. 


\title{
APT LLRF CONTROL SYSTEM MODEL RESULTS •
}

\author{
A. Regan, Los Alamos National Laboratory, LANSCE Division, Los Alamos, NM, USA and \\ C. Ziomek, B\&B Technologies, Albuquerque, NM, USA
}

\section{Abstract}

The low-level RF (LLRF) control system is an essential component of the RF system for the Accelerator Production of Tritium (APT). Requisite for good performance at a reasonable cost is system modeling prior to actual hardware build. Models have been created to help establish the LLRF control system baseline design. These models incorporate common signal processing functions and control functions as well as mixed continuous and discrete-time analysis. Components include klystron saturation curves, waveguide delays, realistic resonant cavity equivalents, and LLRF proportional, integral, and differential (PID) control transfer functions. They predict the performance of the LLRF system in the presence of beam noise, excitation of non-fundamental modes which occur in the superconducting cavities, and pulsed beam situations. This paper will describe the basic model and will present results for a variety of operating scenarios.

\section{MODEL DESCRIPTION}

The functionality of the low-level RF (LLRF) control system for the APT was described in reference 1. In order to best design this system we have relied on computer modeling to predict system response to a variety of inputs during different operational scenarios. The software used is MATRIXX' ${ }^{\top M}$, a graphical modeling and analysis control system program. In MATRIXX'M, common signal-processing and control functions such as transfer functions, limiting, dead band, etc. are all available. It allows both linear and nonlinear functions, as well as mixed continuous and discrete-time analysis (e.g., digital control of a continuous-time plant) and it provides iconic programming (functional blocks, signal flow connectors). We have developed LLRF control system models for a variety of reasons: 1) specification of RF components; 2) verification of system design and performance objectives; 3) optimization of control parameters; and 4) testbed for exploratory control system development. This modeling has been utilized and proven on a number of LLRF control system designs: GTA; AFEL; Boeing's APLE; University of Twente's FEL. It is now being used as a tool for the design of the APT LLRF control system.

The graphical modeling approach allows the model to be built as a combination of "superblocks," each representing the transfer function of its individual components. The overall system model schematic is represented by the top-level block diagram shown in figure 1. Represented as baseband in-phase and quadrature signals, the model includes an ideal current-source beam with noise, a multi-mode accelerator cavity (single gap), a non-linear klystron (with saturation and ripple), waveguide and transmission line delays, and a LLRF controller with feedback and feedforward characteristics. The level of detail available to represent individual components within the RF system (including the accelerating cavity) can be significant. With such a model, we have been able to predict how a particular LLRF control system design will react to a variety of operational scenarios. These include the presence of beam noise, klystron high voltage power supply ripple, and beam pulsing or fault shutdown.

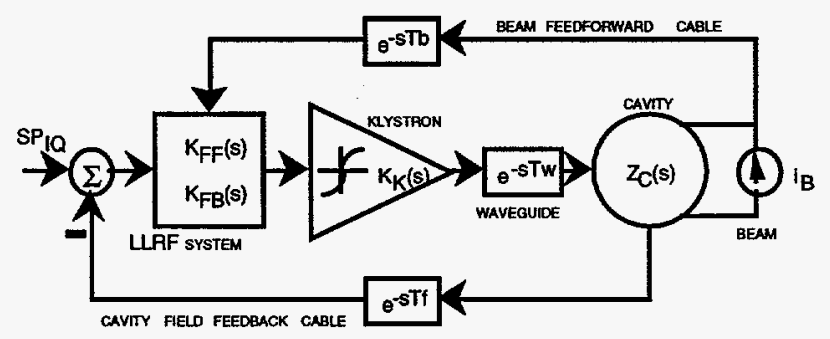

Figure 1. LLRF control system model block diagram.

\section{MODEL RESULTS}

Model analysis provides for certain parameters to be modified depending on the scenario being tested. These external parameters allow easy modification of the core model to investigate different operational cases. Figures 2 through 6 show the results of modeling the case of normal turn-on of the RF into the cavity followed by turn-on of the beam, as well as the case of rapid beam shutdown (due to pulsing or a beam abort).

As seen in figure 2, there is an initial RF turn-on transient in the cavity field and a spike in the field amplitude and phase errors. Figure 2 shows the type of field amplitude and phase errors we can expect for this turn-on/ $\mathrm{CW}$ operation. As seen, the beam turn-on causes a $+1 /-2 \%$ field amplitude error and a $+1 /-0.5^{\circ}$ field phase error. The LLRF control system in this model utilizes just a cavity field feedback signal. An optional beam feedforward feature for tighter control is discussed at the end of this paper.

Figure 3 shows that the klystron saturates until the field gets close to its nominal value when the loop achieves control and the cavity field and klystron forward amplitude settle to their nominal levels (ready for beam). Field errors are now close to zero. Reflected amplitude is

- Work supported by US Department of Energy. 
identical to the forward amplitude without beam, indicating the large mismatch due to strong overcoupling without beam. When the beam is turned on, at $t=500$ $\mu \mathrm{s}$, the klystron forward output increases to compensate for the beam loading and the klystron saturates once more. Again, the cavity field and klystron forward amplitude eventually settle to their nominal levels. Since transients in the RF field will drive modes other than the fundamental in the superconducting cavities, we wanted to study the effects of these other modes. Figures 3 and 4 show the results when we include the two modes nearest to the fundamental. The klystron output does not match perfectly to these other modes in the cavity, and consequently, the reflected signal (figure 3) does not drop to zero as expected when the beam is present. Note the relative magnitudes of the plots in figure 4 . The modes exist and affect the fundamental $700 \mathrm{MHz}$ field thereby causing some slight ripple on the beam to occur, as seen in figure 3. However because the steady-state magnitude of the fundamental is approximately 3400 times that of the $698 \mathrm{MHz}$ mode and even greater than that of the 681 $\mathrm{MHz}$ mode, their effects are fairly minimal. The actual effect on the $700 \mathrm{MHz}$ field by these other modes is indicated in figure 4.
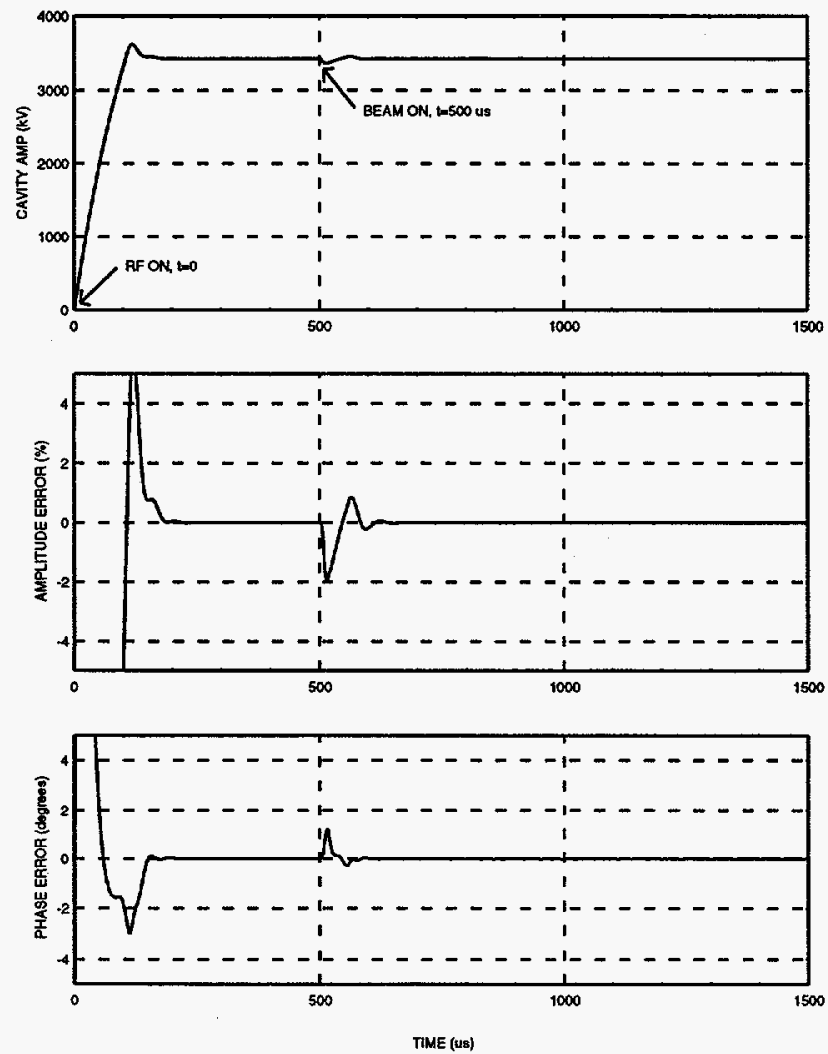

Figure 2. Normal RF and beam turn-on. Cavity field, amplitude and phase errors.
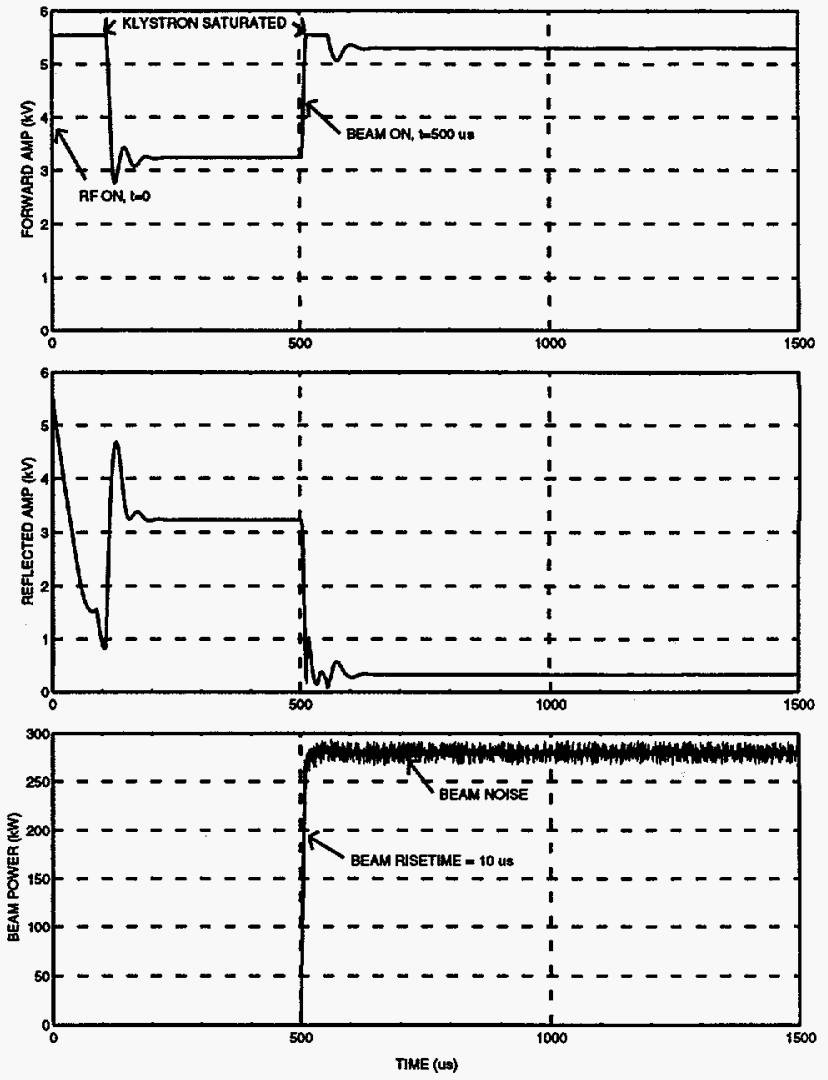

Figure 3. Normal RF and beam turn-on. Forward signal out of klystron, cavity reflected signal, beam power in the cavity.
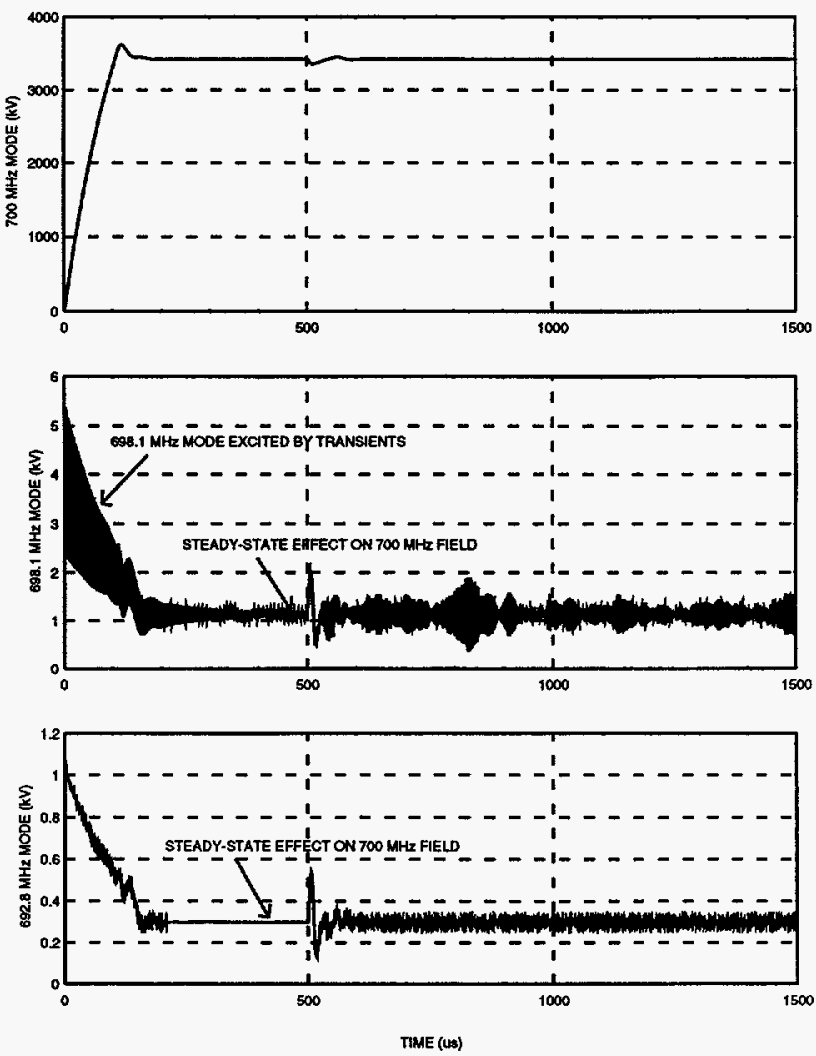

Figure 4. Normal RF and beam turn-on: cavity modes excited. 
Another case investigated with the model was the effect of rapid beam shutdown. This might occur intentionally due to pulsing of the beam, perhaps for cavity conditioning, or unintentionally, due to a beam abort caused by some system error requiring fast beam shutdown. As seen in figures 5 and 6 , without beam feedforward, there is a large transient when the beam is turned off, while the RF remains on. However, this transient is short-lived and should not cause any problems to the cavities.

When the beam is off and the transient passed, the cavity and the klystron return to the states they were in prior to the beam turn-on, and are ready for beam reintroduction. This model indicates that if the beam is turned off, the control system requires it to remain off for at least $50 \mu \mathrm{s}$ before turning it on again in order to reach a controlled steady-state field in the cavity.
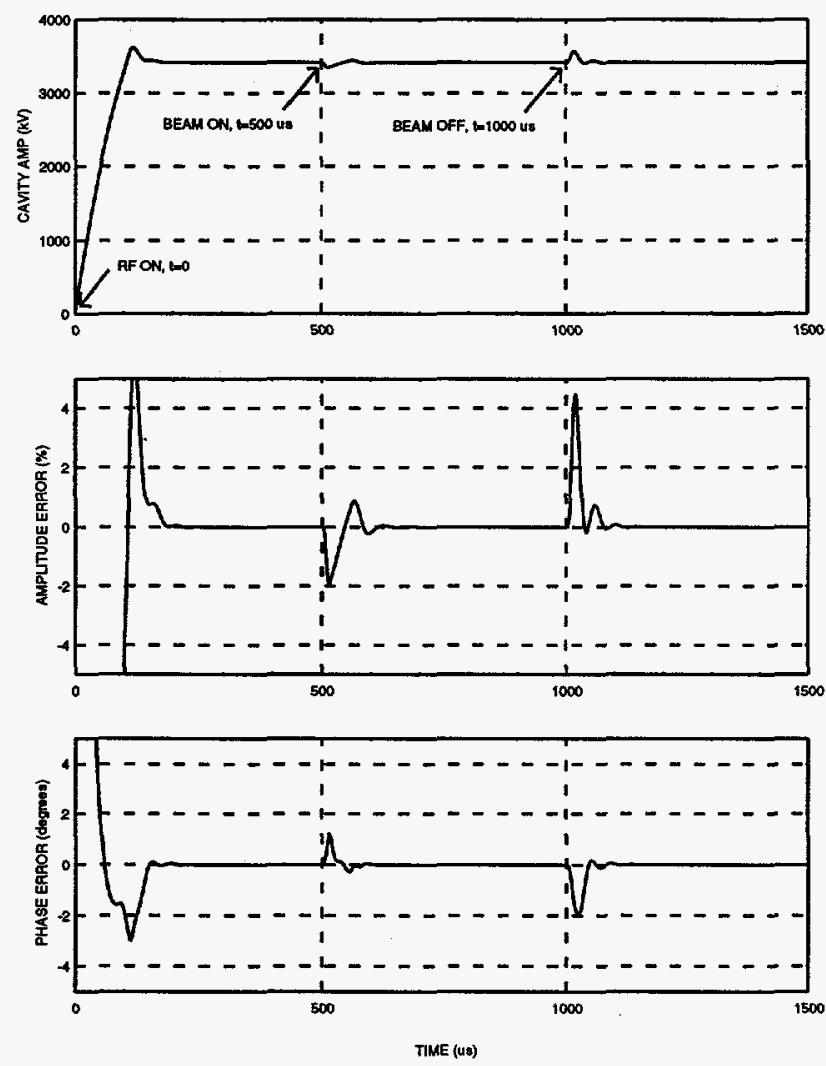

Figure 5. Beam turn-off effect on cavity field, error signals.
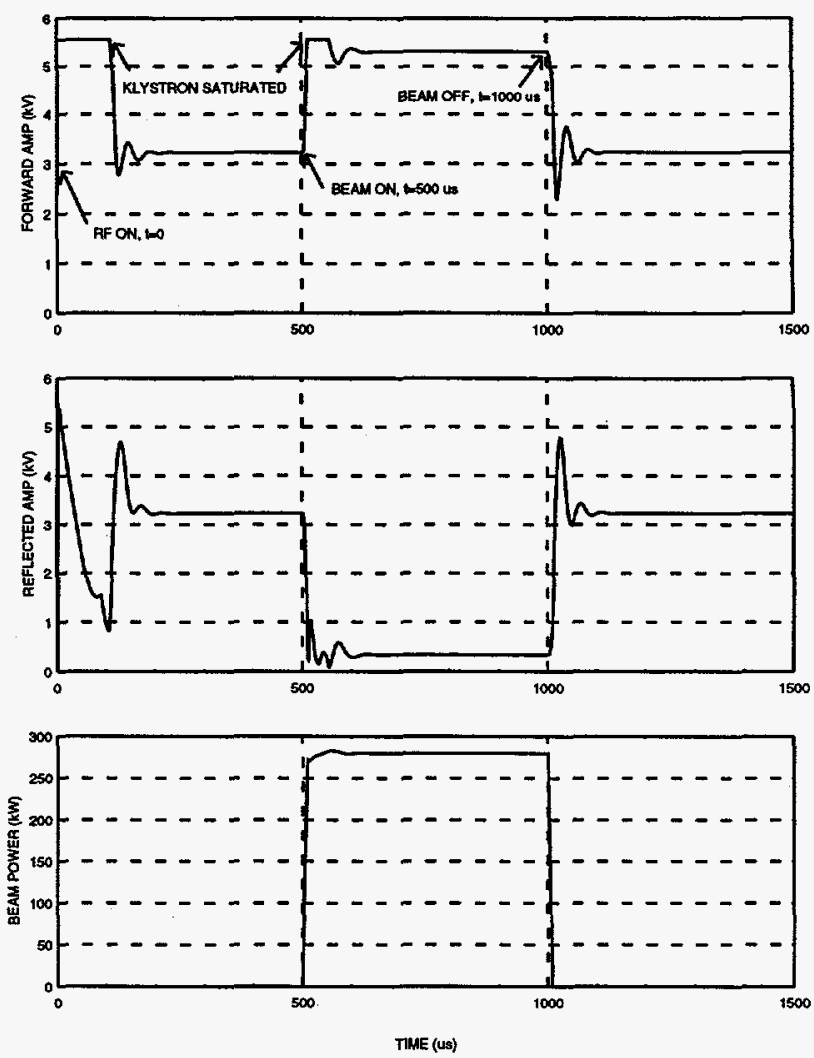

Figure 6. Beam turn-off effect on forward signal out of klystron, cavity reflected signal. Beam power shown.

We have also done some modeling to predict the effectiveness of a beam feedforward feature in the LLRF control system. These models predict that we can reduce the turn-on and turn-off transients that occur in the amplitude and phase errors to $+1 /-0.5 \%$ and $+0.3 /-0.5^{\circ}$ respectively.

\section{CONCLUSIONS}

The modeling effort has been extensive for the APT project. The basic LLRF control system model has been proven on past projects and gives a solid foundation upon which to base our LLRF control system design. We are now in the process of developing VXIbus modules which perform the control functions defined by the models.

\section{REFERENCES}

[1] Regan, A.H., et al, "APT LLRF Control System Functionality and Architecture," Linac96 Proceedings, Geneva, Switzerland, August 1996, pp. 225-227.

[2] Ziomek, C.D., et al, "Low-Level RF Control System Issues for an ADTT Accelerator," ADTT Conference Proceedings, Las Vegas, NV, July, 1994.

[3] Cheo, B.R. and Jachim, S.P., "Dynamic Interactions Between RF Sources and Linac Cavities with Beam Loading," submitted to IEEE Trans. Elec. Dev., 1990. 


\section{DISCLATMER}

Portions of this docament may be illegible in electronic image prodnets. Images are produced from the best available original docoment. 